\title{
Expression of miRNAs and PTEN in endometrial specimens ranging from histologically normal to hyperplasia and endometrial adenocarcinoma
}

\author{
Heejeong Lee ${ }^{1}$, Hyun Joo Choi ${ }^{1}$, Chang Suk Kang ${ }^{1}$, Hee Jin $\mathrm{Lee}^{2}$, Weon Sun Lee ${ }^{2}$ and \\ Chul Soo Park ${ }^{3}$ \\ ${ }^{1}$ Department of Hospital Pathology, The Catholic University of Korea, Seoul, Korea; ${ }^{2}$ Department of \\ Clinical Medicine Research Institute, The Catholic University of Korea, Seoul, Korea and ${ }^{3}$ Department of \\ Internal Medicine, College of Medicine, The Catholic University of Korea, Seoul, Korea
}

\begin{abstract}
We investigated the relationship between frequently deregulated microRNAs (miRNAs) and enodometrial pathology in an attempt to find the most dependable miRNA or combination of miRNAs to identify normal, hyperplastic and malignant endometrial tissues. We also investigated the association between those miRNAs and PTEN status. We measured the expression of six miRNAs (miR-21, 182, 183, 200a, 200c and 205) in 75 formalin-fixed, paraffin-embedded normal, hyperplastic, and malignant endometrial tissue blocks using Taqman-based real-time PCR assays. PTEN loss of expression was assessed in the same endometrial tissues by immunohistochemistry. Expression of five miRNAs (miR-182, 183, 200a, 200c and 205) was significantly higher in endometrial carcinoma (CA) when compared with complex atypical hyperplasia (CAH), simple hyperplasia $(\mathrm{SH})$ and normal endometrial tissue $(P<0.05$, respectively). Considering the likelihood ratio and number of parameters, the composite panel of six miRNAs was the best marker, revealing a sensitivity of $91 \%$ and a specificity of $94 \%$ in differentiating endometrial CA from endometrial hyperplasia or normal endometrium while the individual miRNAs exhibited $64-77 \%$ sensitivity and $66-91 \%$ specificity. Interestingly, in distinguishing endometrial CA from CAH, the composite panel of four miRNAs (miR-182, 183, 200a, 200c) was the best marker, producing $95 \%$ sensitivity and $91 \%$ specificity. The percentage of PTEN loss was significantly higher in endometrial CA compared with SH ( $68 \%$ vs $24 \%, P<0.05)$, and it was also higher in CAH compared with $\mathrm{SH}(71 \%$ vs $24 \%, P<005)$. Aberrant expression of miRNAs and loss of PTEN expression are common in endometrial hyperplasia and CA. They might serve to increase the diagnostic reproducibility and improve discrimination, especially, between CAH and CA by miRNA expression profiles and between simple and complex hyperplasia through PTEN expression patterns. Those expression profiles of biomarkers also might be used to predict the potential for progression from endometrial hyperplasia to invasive CA.

Modern Pathology (2012) 25, 1508-1515; doi:10.1038/modpathol.2012.111; published online 6 July 2012
\end{abstract}

Keywords: endometrial hyperplasia and carcinoma; microRNA; PTEN

Endometrial carcinoma (CA) is the most common female genital tract malignancy in developed countries such as the United States. ${ }^{1}$ According to

Correspondence: Professor CS Park, MD, PhD, Division of Cardiology, Department of Internal Medicine, College of Medicine, The Catholic University, Saint Mary's hospital, 62 Yoidodong, Seoul 150-713, Korea.

E-mail: Charlie@catholic.ac.kr

Received 7 February 2012; revised 8 May 2012; accepted 14 May 2012; published online 6 July 2012 national tumor registry, ${ }^{2,3}$ the incidence of endometrial cancer in Korea also remarkably increased from 1991 to 2005.

Clinicopathologic and epidemiologic studies have supported the malignant potential of endometrial hyperplasia and the concept of continuum of proliferative glandular lesions culminating, in some cases, in CA. ${ }^{4}$ Accurate diagnosis and curative intervention of those precancerous lesions are as important for endometrial cancers as for cervical cancers. However, two factors make classification 
of endometrial hyperplasia suboptimal, such as curettage sampling errors and subjective WHO classification. ${ }^{5}$ Diagnosis of such a precancerous endometrial hyperplasia, especially the criteria for cytologic atypia, which confers a significantly increased risk of progression to $\mathrm{CA},{ }^{6-8}$ is very subjective among pathologists and has shown a low interobserver reproducibility of $<50 \%$ in most studies. ${ }^{5,7}$ Those results prevent the WHObased classification of endometrial hyperplasia to guide patient clinical management and treatment effectively. ${ }^{5}$

MicroRNAs are small noncoding RNAs that have been implicated in tumor development. They regulate target gene expression either by mRNA degradation or by translation repression. ${ }^{9-17}$ In general, each microRNA (miRNA) can regulate up to hundreds of target genes. ${ }^{9,17}$ During tumor development, aberrant expression of miRNAs can either inactivate tumor suppressor genes or activate oncogenes, ${ }^{10-12,14-16,18}$ thereby promoting tumor formation. ${ }^{10-12,14-16,18}$ Because expression of miRNAs is tissue-specific, ${ }^{10}$ detectable in blood ${ }^{19}$ and correlates with clinical cancer behaviors, ${ }^{20}$ miRNAs are potential valuable biomarkers.

Until recently, several studies have identified either upregulated or downregulated miRNAs in endometrial tissue samples. They used the global profiling method m-29 $^{21}$ or the candidate miRNA approach ${ }^{30-36}$ and showed that aberrant expression of miRNAs has been associated with tumor histology, ${ }^{26-28}$ response to steroid therapy ${ }^{24}$ or chemotherapy $^{29}$ and survival. ${ }^{21,22,25}$ However, most studies determined miRNA expression in either normal endometrial tissues or cancer tissues, and the possible miRNA expression in endometrial hyperplasia is largely unknown.

Mutations in PTEN have been found in more than $20 \%$ of hyperplasias, both with and without atypia, and in $30-80 \%$ of endometrial CAs, suggesting that alterations in PTEN occur at a relatively early stage in endometrial tumorigenesis. ${ }^{4}$ Characterization of the complex relationship between miRNAs and the target gene PTEN in endometrial lesions may help further to define some of the molecular pathways that drive carcinogenesis. Thus, we decided to seek for the differential expression of six miRNAs, which have been reported most frequently in endometrial cancers $^{21-23,25,27-29,31,36}$ and PTEN in a variety of endometrial tissues to understand the endometrial carcinogenesis and apply those expression profiles to the pathologic diagnostic field. Those biomarkers would help to increase the diagnostic reproducibility of endometrial hyperplasia and also improve discrimination between complex atypical hyperplasia (CAH) and endometrial CA. Those expression profiles of biomarkers might also be expected to identify those patients with endometrial hyperplasia who have concurrent CA or are at risk of developing it.

\section{Materials and methods}

\section{Endometrial Tissue Samples and Patients}

A total of 75 archived formalin-fixed, paraffinembedded (FFPE) tissue blocks of normal endometrium (10 cases), simple hyperplasia ( $\mathrm{SH}, 21$ cases), CAH (22 cases) and endometrial CA (22 cases) were obtained from the Pathology Department of Bucheon St Mary's Hospital from 1994 to 2004. The patients' age ranged from 29 to 77 years, with a median of 47 years. Of 22 CAs, 16 cases were myometrial-invasive with 15 patients in substage IA, 5 in substage IB and 2 in substage IIIC1, according to the International Federation of Gynecology and Obstetrics standards.

Histologic diagnoses were reviewed by two independent pathologists. Normal tissues were obtained from the patients who had a hysterectomy for benign uterine pathologies such as leiomyoma, adenomyosis or prolapsed uteri. Only tissue blocks with more than $80 \%$ of hyperplasia or CA content were used for this study. For endometrial CAs, we selected only well-differentiated (grade 1), endometrioid-type, endometrial CAs because they are the main targets we should discriminate from CAHs.

\section{RNA Extraction}

Total RNA from FFPE tissues was extracted using RecoverAll Total Nucleic Acid Extraction Kit (Ambion, Texas, USA) following the manufacturer's protocol. RNA concentration and purity were assessed using UV Spectrophotometer.

\section{Reverse Transcription- and Quantitative Real-Time PCRs}

Reverse transcription and quantitative real-time PCRs were performed for the following miRNAs: miR-205, miR-183, miR-200c, miR-200a, miR-182 and miR-21 using the MicroRNA TaqMan Reverse Transcription Kit and the TaqMan MicroRNA Assays in triplicate (Applied Biosystems, Foster City, CA, USA). U6 small nuclear 2 (RNU6b) was used to normalize input total small RNA. Absolute quantification for each miRNA as well as RNU6b was performed using a standard curve generated by serial dilution of reverse transcribed total RNA extracted from VK2 cells, and expression of each miRNA was presented as the ratio between miRNA and RNU6b (RQ).

\section{Immunohistochemical Analysis of PTEN Loss of Expression}

Material was available from all cases of endometrial tissues for immunohistochemical analysis of PTEN loss of expression. Four-micron sections of FFPE tissue were cut and placed on Superfrost Plus microscope slides (VWR, San Francisco, CA, USA). 
The tissue sections were deparaffinized and rehydrated through graded alcohols. Endogenous peroxidase activity was blocked by incubation in $3 \%$ $\mathrm{H}_{2} \mathrm{O}_{2}$. Antigen retrieval was carried out with $0.01 \mathrm{M}$ citrate buffer $\mathrm{pH} 6.0$ and microwave heat induction. Approximately $100 \mu \mathrm{l}$ of the primary rabbit monoclonal antibody (diluted 1:100; Cell Signaling Technology, clone138G6, Danvers, MA, USA) or antibody diluents lacking the primary antibody was applied to each slide. The slides were washed, and a biotinylated anti-rabbit antibody (diluted 1:500; Vector Laboratories, Burlingame, CA, USA) was applied. After a second wash, the avidin-biotin-peroxidase complex (Vector Laboratories) was applied. Color development was accomplished by incubation in diaminobenzidine with $3 \% \mathrm{H}_{2} \mathrm{O}_{2}$ as a substrate, and nickel chloride enhancement. The slides were counterstained with methyl green, dehydrated through graded alcohols, cleared in xylene and coverslipped with permanent mounting media. All cases were reviewed and interpreted without the knowledge of other laboratory or clinical results. Individual glands were scored as PTEN-null when the signal was absent in the nuclear and cytoplasmic compartments of most cells in that gland. Because the burden of PTEN-null glands in general remains less than $2-5 \%$ until clonal expansion into an EIN lesion, ${ }^{37}$ specimens were scored as PTEN-positive if more than $95 \%$ of endometrial glands expressed PTEN with 5\% and less PTEN-null glands, heterogenous with 6-94\% PTEN-null glands and PTENnegative with more than 95\% PTEN-null glands. Immunohistochemical loss of PTEN included specimens scored as negative and heterogeneous according to previous reported methods. ${ }^{38,39}$

\section{Statistical Analysis}

Kruskal-Wallis test was used to compare the RQ of miRNA expression with respect to pathological findings of patients. Pathologic diagnosis was used as a standard reference. The receiver-operator characteristic curve was constructed and the cutoff values of each miRNA and the composite panel of miRNAs that produced high overall accuracy were selected. The sensitivity and specificity in differentiating CA from other tissues and CA from CAH were calculated for each cutoff. The area under receiver-operating characteristic curve (AUC) was also estimated. To determine the best composite panel of miRNAs, we used Akaike's Information Criteria, which determines the best fitting model based on the likelihood ratio test and the number of model parameters. ${ }^{40}$ The difference of PTEN expression between the pathologic groups was tested with $\chi^{2}$ test, and the difference of miRNA expressions between PTEN loss and expressor specimens was tested with Mann-Whitney test. A two-sided 0.05 test level determined statistical significance for all analyses.

\section{Results}

\section{MicroRNA Expression in Clinical Samples}

Expression levels of five miRNAs (miR-182, 183, 200a, 200c and 205) were significantly higher in endometrial CA when compared with $\mathrm{CAH}, \mathrm{SH}$ and normal endometrial tissue $(P<0.05$, respectively; Figure 1). Expression level of miRNA-21 was moderately elevated in endometrial CA compared with CAH $(P<0.1)$. No miRNA expression level was significantly different among normal endometrium, $\mathrm{SH}$ and CAH.

\section{MicroRNAs Panel Optimized as Biomarkers for Endometrial Carcinoma}

The individual microRNAs exhibited AUC values of 0.665-0.904 in distinguishing endometrial CA from
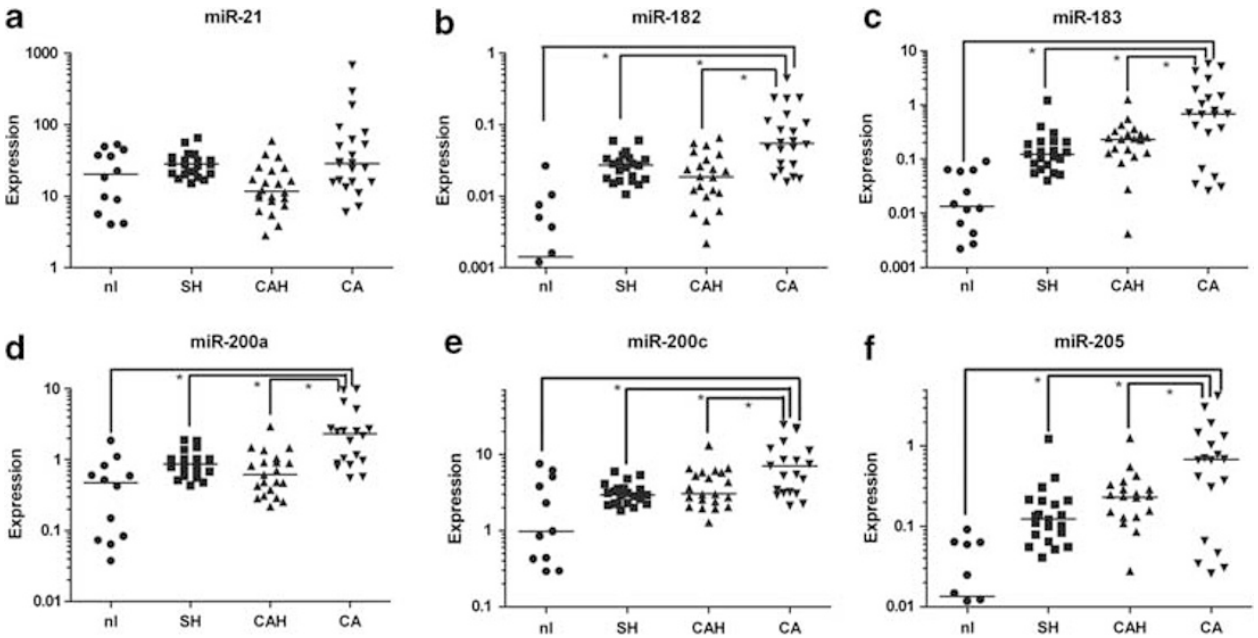

Figure 1 (a-f) The expression levels of miRNA-182, 183, 200a, 200c and 205 were significantly higher in endometrial carcinoma (CA) compared with normal endometrium (nl), simple hyperplasia (SH) or complex hyperplasia. The horizontal line indicates the median value. ${ }^{*} P<0.05$, endometrial $\mathrm{CA} v s \mathrm{nl}, \mathrm{SH}$, complex atypical hyperplasia (CAH), respectively. 
endometrial hyperplasia or normal endometrium, revealing $64-77 \%$ sensitivity and 66-91\% specificity (all $P<0.05$; Table 1). In discriminating endometrial CAs from other tissues, the composite panel of all six miRNAs was determined to be the best fitting model. The composite panel of six miRNAs produced 0.961 AUC that was higher than any other individual gene used alone $(P<0.05)$. Given a specificity of $94 \%$, the composite panel revealed a sensitivity of $91 \%$ in differentiating endometrial CA cases from hyperplasia or normal cases (Figure 2).

When discriminating endometrial CA from CAH, the individual microRNAs exhibited AUC values of 0.760-0.896, revealing 67-82\% sensitivity and 63$77 \%$ specificity (all $P<0.05$; Table 2). The best fitting panel consisted of four miRNAs (miR182, 183, 200a and 200c). This panel exhibited AUC values of $0.957,95 \%$ sensitivity and $91 \%$ specificity in distinguishing endometrial CA from CAH (Figure 3).

Table 1 Capability of the miRNAs to discriminate endometrial carcinoma from endometrial hyperplasia or normal endometrium

\begin{tabular}{lccc}
\hline miRNAs & AUC $($ SE) & $\begin{array}{c}\text { Sensitivity } \\
(\%)\end{array}$ & $\begin{array}{c}\text { Specificity } \\
(\%)\end{array}$ \\
\hline miR-21 & $0.665(0.074)$ & 67 & 66 \\
miR-182 & $0.855(0.048)$ & 64 & 91 \\
miR-183 & $0.904(0.037)$ & 77 & 80 \\
miR-200a & $0.847(0.050)$ & 76 & 72 \\
miR-200c & $0.790(0.060)$ & 67 & 74 \\
miR-205 & $0.799(0.070)$ & 77 & 86 \\
6miRNAs & $0.961(0.025)$ & 91 & 94 \\
\hline
\end{tabular}

Abbreviations: AUC, the area under receive--operating characteristics curve; SE, standard error.

All $P<0.05$.

\section{PTEN Status in Endometrial Tissue Samples and Association with MicroRNA Expression}

Loss of PTEN expression was determined in the same endometrial tissues by immunohistochemical analysis. Altered PTEN expression was noted in 2 of 10 normal endometrium (20\%), 5 of $21 \mathrm{SH}(24 \%), 15$ of $21 \mathrm{CAH}(71 \%)$ and 15 of 22 endometrial CA (68\%). Among PTEN loss specimens, PTEN heterogenous type was more frequently detected in normal and $\mathrm{SH}$ compared with CAH and CA (86\% vs $10 \%$; Figure 4). The percentage of PTEN loss (PTENnegative and heterogeneous cases) was significantly higher in endometrial CA compared with $\mathrm{SH}$ (68\% vs 24\%, $P<0.05)$; it was also higher in $\mathrm{CAH}$ compared with SH (71\% vs 24\%, $P<0.05$; Figure 5). Expression of miR-200c was significantly higher in PTEN-negative tissue compared with PTEN-positive tissue $(P<0.05)$. Expression of miR-21 and miR-183 did not show a significant correlation with PTEN expression patterns.

Table 2 Capability of the miRNAs to discriminate endometrial carcinoma from complex atypical hyperplasia

\begin{tabular}{lccc}
\hline MiRNAs & AUC(SE) & $\begin{array}{c}\text { Sensitivity } \\
(\%)\end{array}$ & $\begin{array}{c}\text { Specificity } \\
(\%)\end{array}$ \\
\hline miR-21 & $0.771(0.072)$ & 81 & 63 \\
miR-182 & $0.850(0.057)$ & 81 & 73 \\
miR-183 & $0.896(0.046)$ & 82 & 73 \\
miR-200a & $0.855(0.056)$ & 77 & 73 \\
miR-200c & $0.760(0.073)$ & 67 & 64 \\
miR-205 & $0.773(0.090)$ & 77 & 77 \\
4miRNAs & $0.957(0.032)$ & 95 & 91 \\
\hline
\end{tabular}

Abbreviations: AUC, the area under receiver-operating characteristics curve; SE, standard error.

All $P<0.05$
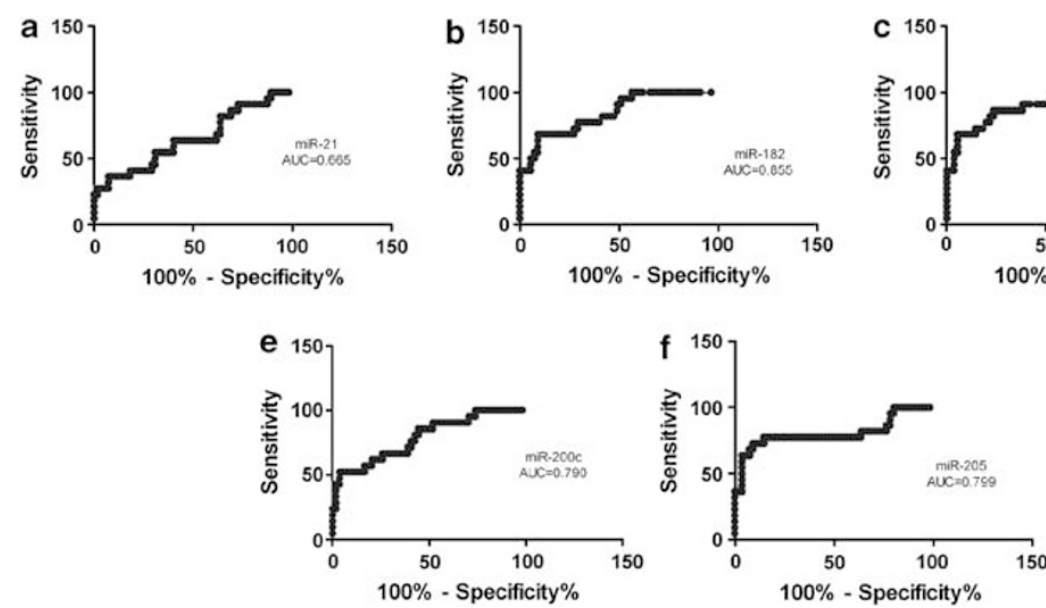
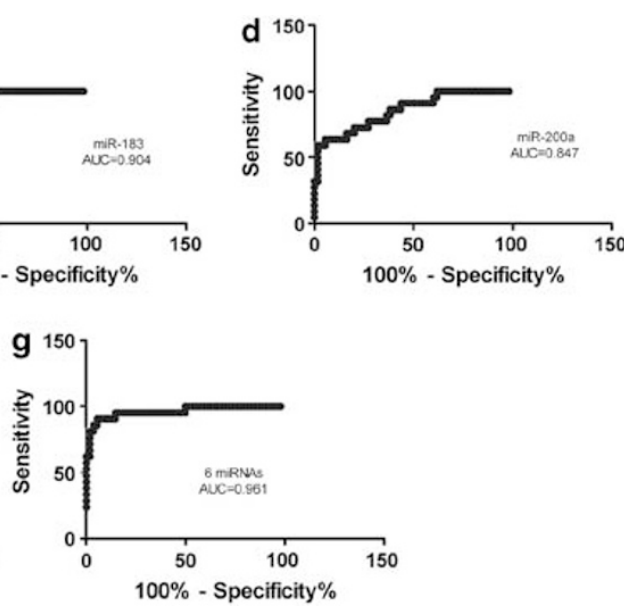

Figure 2 (a-g) Receiver-operator characteristic (ROC) curve analysis of expression levels of miRNAs. The area under the ROC curve (AUC) for each miRNA indicates its accuracy in differentiating endometrial carcinoma from endometrial hyperplasia or normal endometrium. All $P$-values $<0.05$. 

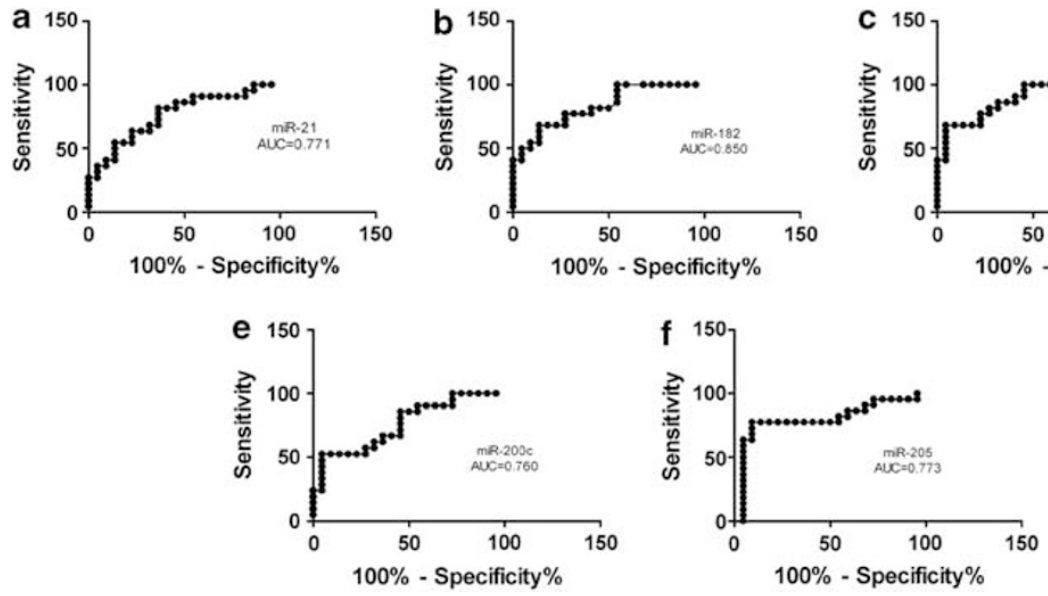
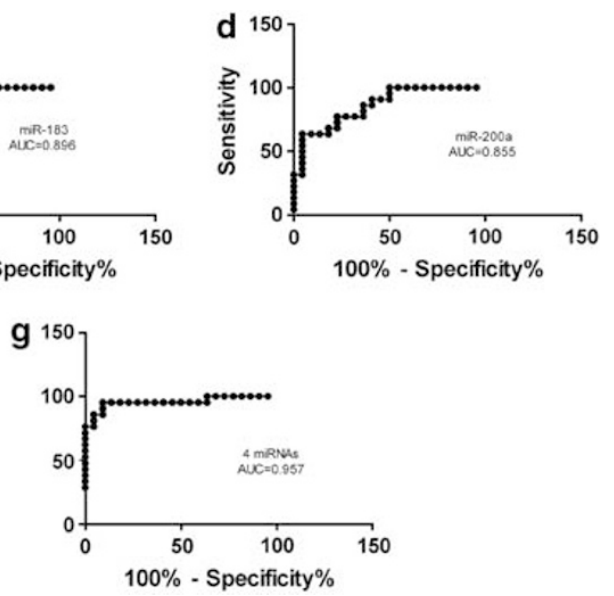

Figure 3 (a-g) Receiver-operator characteristic (ROC) curve analysis of expression levels of miRNAs. The area under the ROC curve (AUC) for each miRNA indicates its accuracy in differentiating endometrial carcinoma from complex atypical hyperplasia. All $P<0.05$.
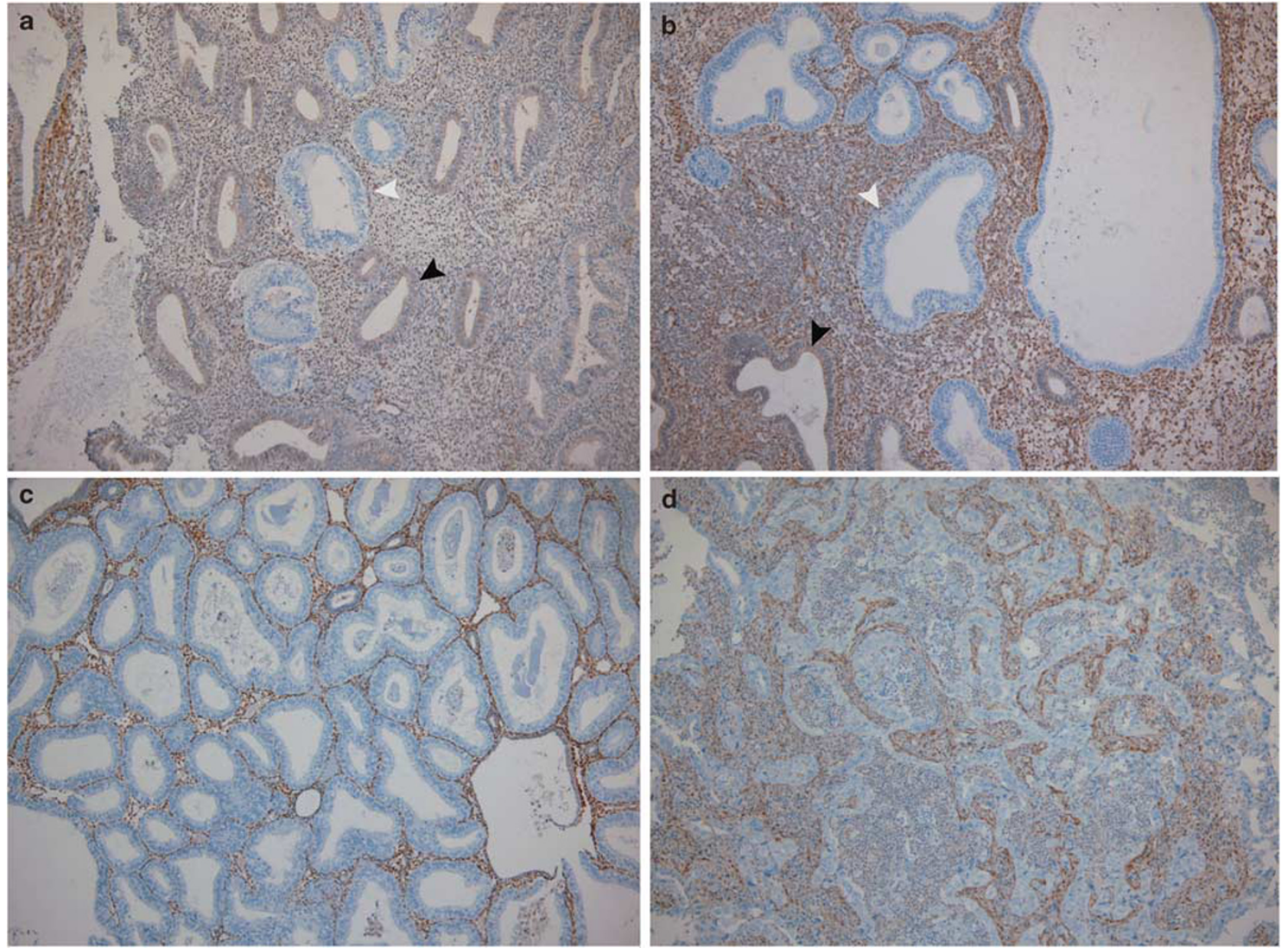

Figure 4 (a-d) Immunohistochemical loss of PTEN in (a) normal endometrium (PTEN loss, heterogeneous); (b) simple hyperplasia (PTEN loss, heterogeneous); (c) complex atypical hyperplasia (PTEN loss, -negative); and (d) endometrial carcinoma (PTEN loss, -negative). The white arrowhead indicates representative PTEN-null gland, and the black arrowhead indicates representative PTENexpressor gland.

\section{Discussion}

In the present study, we determined the expression of the six miRNAs most frequently reported in endometrial cancers, ${ }^{21-23,25,27-29,31,36}$ correlated with pathologic findings as well as PTEN tumor suppressor expression. Recently, several studies have noted associations between microRNA expressions 


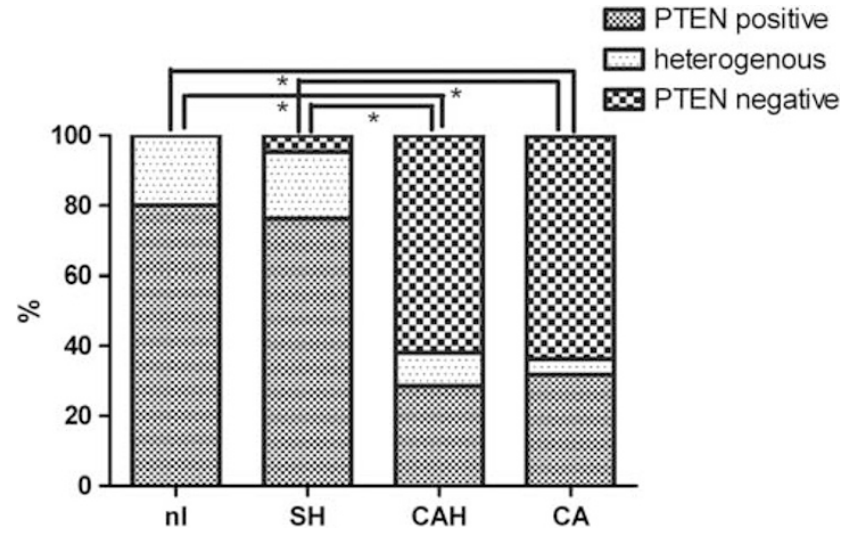

Figure 5 Differential expression of PTEN in endometrial tissues. ${ }^{*} P<0.05$, PETN loss (PTEN-negative + heterogeneous) vs PTENpositive; endometrial carcinoma (CA) or complex atypical hyperplasia (CAH) vs normal endometrium (nl) or simple hyperplasia (SH), respectively.

and endometrial CAs. Most frequently, miR$205,,^{21,23,25,27,28} 200 \mathrm{c},,^{21,25,28,29,31} 200 \mathrm{a},{ }^{25,27-29} 182,,^{25,27,36}$ and $183,{ }^{21,25,27,36}$ were reported to be upregulated in endometrial CAs including cancer cell lines compared with normal endometrial controls, especially in early-stage and well-differentiated cancers. A few of the most recent studies reported upregulated miRNA-200 family in endometrioid endometrial CA wit ${ }^{29}$ showing that all members of miR-200 family (200a, 200b, 200c, 149, 429) were upregulated in endometrioid endometrial CA compared with the levels in normal endometrial tissues. However, those results are less informative from a pathological standpoint because it is not so difficult to discriminate between cancer and normal endometrial tissues. We thought we needed more practical biomarkers for differential diagnosis between CAH and welldifferentiated endometrioid-type endometrial CA or between simple and complex hyperplasia. It is those lesions that we pathologists find it particularly difficult to make a diagnosis in daily routine practice. Although Snowdon et $a l^{28}$ showed that 43 dysregulated miRNAs in endometrioid endometrial CA and CAH compared with normal controls from microarray study, they did not suggest any practically helpful panels of miRNAs to discriminate among those three lesions.

Surprisingly, we detected significant differences between normal endometriun or hyperplastic tissue and endometrial CA in five out of six miRNAs. The higher expression of miR-182, 183, 200a, 200c and 205 was noted in endometrial CA when compared with $\mathrm{CAH}, \mathrm{SH}$ and normal endometrial tissue. All of the six miRNAs exhibited statistically discriminating power between endometrial CA and other tissue types, with miR-183, in particular, showing higher than $80 \%$ sensitivity and specificity. We also found that a composite panel of six miRNAs was the statistically best biomarker.

From a clinical perspective, it is particularly difficult to differentiate between $\mathrm{CAH}$ and endome- trial CA in some cases, and correct pathologic diagnosis is critical in treating these patients. In our study, five out of six miRNAs showed higher expression in endometrial CA compared with CAHs. Based on AUC analysis, individual miRNAs were proven to be substantially informative. However, the composite panel of four miRNAs consisting of miR-182, 183, 200a and 200c was the best combined biomarker to differentiate endometrial CA from CAH.

Considering the high sensitivity and specificity above $90 \%$, it might be used as a supplementary diagnostic tool in complicated cases.

PTEN is the confirmed target of miR-21,41 and predicted target of miR-200c and 183. Predicted targets were examined with http://microrna.sanger. ac.uk/sequences. ${ }^{21}$ In a few studies, PTEN immunohistochemical expression patterns have been reported to have utility as a diagnostic marker for differentiating normal, hyperplastic and neoplastic endometrium. ${ }^{8,42,43}$ Pavlakis et al $^{44}$ suggested that PTEN-loss and nuclear atypia of EIN in endometrial biopsies can predict the existence of a concurrent endometrial CA. However, some other studies have failed to show that lack of PTEN expression has the specificity and reproducibility to be clinically useful as a biomarker. ${ }^{7,45,46}$ Thus, we decided to ascertain the role of PTEN tumor suppressor gene in endometrial tumors and the relationship with miRNA alterations including specific miRNAs, which were considered to target PTEN tumor suppressor gene. ${ }^{9,21}$

In the present study, the percentage of PTEN loss including PTEN-negative and heterogeneous cases was significantly higher in endometrial CA and CAH compared with SH and normal endometrium. Interestingly, miR-200c was significantly upregulated in various PTEN-negative endometrial tissues. However, based on our data that showed a discrepancy between PTEN loss and miR-200c expression in CAH, another mechanism or miRNAs might be involved in the process of PTEN loss in the endometrial carcinogenesis pathway. Although PTEN is the confirmed target of miRNA-21 and miRNA-21 is considered to be involved in the pathogenesis of endometriosis ${ }^{24,47}$ and the estrogen receptor network, ${ }^{36}$ miRNA-21 does not seem to be involved in the endometrial tumorigenesis in our study.

The results of our study suggest that several miRNAs (miR-182, 183, 200a, 200c and 205) are involved in progression from complex atypical endometrial hyperplasia to CA, whereas PTEN has a role in the progression from $\mathrm{SH}$ to $\mathrm{CAH}$. Thus, using those expression profiles in the diagnostic field, we could estimate the malignant potential of the endometrial tissue samples that are histopathologically controversial. That is, the normalized ratio of a real-time PCR result of miRNAs might be used to discriminate between $\mathrm{CAH}$ and well-differentiated endometrial CAs, while PTEN loss of expression pattern seems useful for differential diagnosis between simple and CAH. Furthermore, although 
endometrial tissue samples are hyperplasia, or less histopathologically, if the upregulated miRNA expression profile matches that of endometrial CA, we may assume that the possibility of concurrent endometrial CA or high risk of progression to CA is very likely. However, future studies with a test set, where we could try using our panel on a set of unknown cases to see whether they were able to pick up any unrecognized cases of endometrial adenocarcinoma are warranted to determine whether this interaction truly implies and stratifies risks. If the panel is able to detect cases that are missed by surgical pathologists, who then have the opportunity to correct their diagnoses before signing the case out, it could be really valuable, and could be applied in difficult cases.

There has been no report on applying miRNA and PTEN expression profiles for histopathologic differential diagnosis among simple, $\mathrm{CAH}$ and endometrial CA. It is hoped that expression profiles of those biomarkers could help us choose one of several categories of endometrial lesions with more confidence, particularly when distinguishing between $\mathrm{CAH}$ and well-differentiated endometrial CA.

\section{Acknowledgements}

This work was supported by grants from the Institute of Clinical Medicine Research of Bucheon St Mary's Hospital. Informed consent was obtained according to procedures approved by the Human Subjects Committee of the Catholic University of Korea.

\section{Disclosure/conflict of interest}

The authors declare no conflict of interest.

\section{References}

1 Jemal A, Siegel R, Xu J, et al. Cancer statistics, 2010. CA Cancer J Clin 2010;60:277-300.

2 Department of Gynecologic Tumor, Korean Society of Obstetrics and Gynecology. Annual report of gynecologic cancer registry program in Korea for 2001. Korean J Obstet Gynecol 2003;46:1849-1887.

3 Won YJ, Sung J, Jung KW, et al. Nationwide cancer incidence in Korea, 2003-2005. Cancer Res Treat 2009;41:122-131.

4 Cotran RS, Kumar V, Robbins SL. Robbins Pathologic Basis of Disease 8th edn. WB Saunders: Philadelphia, 2009, pp 1030-1034.

5 Lacey Jr JV, Chia VM. Endometrial hyperplasia and the risk of progression to carcinoma. Maturitas 2009;63: 39-44.

6 Kurman RJ. Blaustein's Pathology of the Female Genital Tract 5th edn. Springer-Verlag: New York, 2002, pp 467-484

7 Kurman RJ, McConnell TG. Precursors of endometrial and ovarian carcinoma. Virchows Arch 2010;456:1-12.
8 Allison KH, Tenpenny E, Reed SD, et al. Immunohistochemical markers in endometrial hyperplasia: is there a panel with promise? A review. Appl Immunohistochem Mol Morphol 2008;16:329-343.

9 Pan Q, Chegini N. MicroRNA signature and regulatory functions in the endometrium during normal and disease states. Semin Reprod Med 2008;26:479-493.

10 Zhang W, Dahlberg JE, Tam W. MicroRNAs in tumorigenesis: a primer. Am J Pathol 2007;171:728-738.

11 Corney DC, Nikitin AY. MicroRNA and ovarian cancer. Histol Histopathol 2008;23:1161-1169.

12 Zhang L, Volinia S, Bonome T, et al. Genomic and epigenetic alterations deregulate microRNA expression in human epithelial ovarian cancer. Proc Natl Acad Sci USA 2008;105:7004-7009.

13 Flavin RJ, Smyth PC, Finn SP, et al. Altered eIF6 and Dicer expression is associated with clinicopathological features in ovarian serous carcinoma patients. Mod Pathol 2008;21:676-684.

14 Giannakakis A, Coukos G, Hatzigeorgiou A, et al. miRNA genetic alterations in human cancers. Expert Opin Biol Ther 2007;7:1375-1386.

15 Yang N, Coukos G, Zhang L. MicroRNA epigenetic alterations in human cancer: one step forward in diagnosis and treatment. Int J Cancer 2008;122:963-968.

16 Slack FJ, Weidhaas JB. MicroRNA in cancer prognosis. N Engl J Med 2008;359:2720-2722.

17 Hede K. Small RNAs are raising big expectations. J Natl Cancer Inst 2009;101:840-841.

18 Deng S, Calin GA, Croce CM, et al. Mechanisms of microRNA deregulation in human cancer. Cell Cycle 2008;7:2643-2646.

19 Resnick KE, Alder H, Hagan JP, et al. The detection of differentially expressed microRNAs from the serum of ovarian cancer patients using a novel real-time PCR platform. Gynecol Oncol 2009;112:55-59.

20 Yu SL, Chen HY, Chang GC, et al. MicroRNA signature predicts survival and relapse in lung cancer. Cancer Cell 2008;13:48-57.

21 Cohn DE, Fabbri M, Valeri N, et al. Comprehensive miRNA profiling of surgically staged endometrial cancer. Am J Obstet Gynecol 2010;202:656.e1-656.e8.

22 Hiroki E, Akahira J, Suzuki F, et al. Changes in microRNA expression levels correlate with clinicopathological features and prognoses in endometrial serous adenocarcinomas. Cancer Sci 2010;101:241-249.

$23 \mathrm{Wu}$ W, Lin Z, Zhuang Z, et al. Expression profile of mammalian microRNAs in endometrioid adenocarcinoma. Eur J Cancer Prev 2009;18:50-55.

24 Pan Q, Luo X, Toloubeydokhti T, et al. The expression profile of micro-RNA in endometrium and endometriosis and the influence of ovarian steroids on their expression. Mol Hum Reprod 2007;13:797-806.

25 Chung TK, Cheung TH, Huen NY, et al. Dysregulated microRNAs and their predicted targets associated with endometrioid endometrial adenocarcinoma in Hong Kong women. Int J Cancer 2009;124:1358-1365.

26 Boren T, Xiong Y, Hakam A, et al. MicroRNAs and their target messenger RNAs associated with endometrial carcinogenesis. Gynecol Oncol 2008;110: 206-215.

27 Ratner ES, Tuck D, Richter C, et al. MicroRNA signatures differentiate uterine cancer tumor subtypes. Gynecol Oncol 2010;118:251-257.

28 Snowdon J, Zhang X, Childs T, et al. The microRNA200 family is upregulated in endometrial carcinoma. PLoS One 2011;6:e22828. 
29 Lee JW, Park YA, Choi JJ, et al. The expression of the miRNA-200 family in endometrial endometrioid carcinoma. Gynecol Oncol 2011;120:56-62.

30 Aprelikova O, Yu X, Palla J, et al. The role of miR-31 and its target gene SATB2 in cancer-associated fibroblasts. Cell Cycle 2010;9:4387-4398.

31 Cochrane DR, Spoelstra NS, Howe EN, et al. MicroRNA-200c mitigates invasiveness and restores sensitivity to microtubule-targeting chemotherapeutic agents. Mol Cancer Ther 2009;8:1055-1066.

$32 \mathrm{Wu}$ J, Qian J, Li C, et al. miR-129 regulates cell proliferation by downregulating Cdk6 expression. Cell Cycle 2010;9:1809-1818.

33 Valeri N, Gasparini P, Fabbri M, et al. Modulation of mismatch repair and genomic stability by miR-155. Proc Natl Acad Sci USA 2010;107: 6982-6987.

34 Shen R, Pan S, Qi S, et al. Epigenetic repression of microRNA-129-2 leads to overexpression of SOX4 in gastric cancer. Biochem Biophys Res Commun 2010;394:1047-1052.

35 Daikoku T, Hirota Y, Tranguch S, et al. Conditional loss of uterine Pten unfailingly and rapidly induces endometrial cancer in mice. Cancer Res 2008;68: 5619-5627.

36 Myatt SS, Wang J, Monteiro LJ, et al. Definition of microRNAs that repress expression of the tumor suppressor gene FOXO1 in endometrial cancer. Cancer Res 2010;70:367-377.

37 Zheng W, Baker HE, Mutter GL. Involution of PTENnull endometrial glands with progestin therapy. Gynecol Oncol 2004;92:1008-1013.

38 Djordjevic B, Hennessy BT, Li J, et al. Clinical assessment of PTEN loss in endometrial carcinoma: immunohistochemistry outperforms gene sequencing. Mod Pathol 2012;25:699-708.

39 Garg K, Broaddus RR, Soslow RA, et al. Pathologic scoring of PTEN immunohistochemistry in endometrial carcinoma is highly reproducible. Int J Gynecol Pathol 2012;31:48-56.

40 Akaike H. A new look at the statistical model identification. IEEE Trans Automat Contr 1974;19:716-723.

41 Klinge CM. Estrogen regulation of MicroRNA expression. Curr Genomics 2009;10:169-183.

42 Sarmadi S, Izadi-Mood N, Sotoudeh K, et al. Altered PTEN expression; a diagnostic marker for differentiating normal, hyperplastic and neoplastic endometrium. Diagn Pathol 2009;4:41.

43 Tantbirojn P, Triratanachat S, Trivijitsilp $\mathrm{P}$, et al. Detection of PTEN immunoreactivity in endometrial hyperplasia and adenocarcinoma. Med Assoc Thai 2008;91:1161-1165.

44 Pavlakis K, Messini I, Vrekoussis T, et al. PTEN-loss and nuclear atypia of EIN in endometrial biopsies can predict the existence of a concurrent endometrial carcinoma. Gynecol Oncol 2010;119:516-519.

45 Xiong Y, Xiong YY, Zhou YF. Expression and significance of beta-catenin, Glut-1 and PTEN in proliferative endometrium, endometrial intraepithelial neoplasia and endometrioid adenocarcinoma. Eur J Gynaecol Oncol 2010;31:160-164.

46 Lacey Jr JV, Mutter GL, Ronnett BM, et al. PTEN expression in endometrial biopsies as a marker of progression to endometrial carcinoma. Cancer Res 2008;68:6014-6020.

47 Aghajanova L, Giudice LC. Molecular evidence for differences in endometrium in severe versus mild endometriosis. Reprod Sci 2011;18:229-251. 